\title{
Tat-Fused Recombinant Human SAG Prevents Dopaminergic Neurodegeneration in a MPTP-Induced Parkinson's Disease Model
}

\author{
Eun Jeong Sohn ${ }^{1,5}$, Min Jea Shin ${ }^{1,5}$, Dae Won Kim ${ }^{2,5}$, Eun Hee Ahn ${ }^{1}$, Hyo Sang Jo ${ }^{1}$, Duk-Soo Kim ${ }^{3}$, \\ Sung-Woo Cho ${ }^{4}$, Kyu Hyung Han ${ }^{1}$, Jinseu Park', Won Sik Eum ${ }^{1}$, Hyun Sook Hwang ${ }^{1, *}$, and Soo Young Choi ${ }^{1, *}$
}

\begin{abstract}
Excessive reactive oxygen species (ROS) generated from abnormal cellular process lead to various human diseases such as inflammation, ischemia, and Parkinson's disease (PD). Sensitive to apoptosis gene (SAG), a RING-FINGER protein, has anti-apoptotic activity and anti-oxidant activity. In this study, we investigate whether Tat-SAG, fused with a Tat domain, could protect SH-SY5Y neuroblastoma cells against 1-methyl-4-phenylpyridinium $\left(\mathrm{MPP}^{+}\right)$and dopaminergic (DA) neurons in the substantia nigra (SN) against 1methyl-4-phenyl-1,2,3,6-tetra-hydropyridine (MPTP) toxicity. Western blot and immunohistochemical analysis showed that, unlike SAG, Tat-SAG transduced efficiently into SHSY5Y cells and into the brain, respectively. Tat-SAG remarkably suppressed ROS generation, DNA damage, and the progression of apoptosis, caused by MPP ${ }^{+}$in SH-SY5Y cells. Also, immunohistochemical data using a tyrosine hydroxylase antibody and cresyl violet staining demonstrated that Tat-SAG obviously protected DA neurons in the SN against MPTP toxicity in a PD mouse model. TatSAG-treated mice showed significant enhanced motor activities, compared to SAG- or Tat-treated mice. Therefore, our results suggest that Tat-SAG has potential as a therapeutic agent against ROS-related diseases such as PD.
\end{abstract}

\footnotetext{
${ }^{1}$ Department of Biomedical Science and Research Institute of Bioscience and Biotechnology, Hallym University, Chunchon 200-702, Korea, ${ }^{2}$ Department of Biochemistry and Molecular Biology, Research Institute of Oral Sciences, College of Dentistry, Kangnung-Wonju National University, Gangneung 210-702, Korea, ${ }^{3}$ Department of Anatomy, College of Medicine, Soonchunhyang University, Cheonan 330-090, Korea, ${ }^{4}$ Department of Biochemistry and Molecular Biology, University of Ulsan College of Medicine, Seoul 138-736, Korea, ${ }^{5}$ These authors contributed equally to this work.

*Correspondence: sychoi@hallym.ac.kr (SYC); wazzup@ hallym.ac.kr (HSH)
}

Received 30 October, 2013; revised 18 January, 2014; accepted 27 January, 2014; published online 13 March, 2014

Keywords: dopaminergic neuron, Parkinson's disease, protein transduction domain, reactive oxygen species, sensitive to apoptosis gene

\section{INTRODUCTION}

Reactive oxygen species (ROS) are generated during many cellular processes including catalysis and energy production (Bae et al., 2011; Zhu et al., 2004). In addition, abruptly overproduced ROS cause DNA damage, lipid peroxidation, cell death, and many pathological disorders such as inflammation, ischemia, diabetes, and Parkinson's disease (PD) (Ramalingam and Kim, 2012). However, defense systems in cells remove excessively produced ROS and maintain the cellular balance of ROS (Ramalingam and Kim, 2012). PD, a neurodegenerative disease, is characterized by a progressive loss of dopaminergic (DA) neurons in the substantia nigra (SN) and an accumulation of Lewy bodies (Chinta and Andersen, 2008; Dawson and Dawson, 2003). DA neurons are associated with motor functions and loss of these neurons in PD patients could cause typical symptoms such as tremor, bradykinesia, rigidity, and akinesia (Miller et al., 2009).

Sensitive to apoptosis gene (SAG), a member of the zinc RING finger protein family, is found in both the cytoplasm and nucleus of the cell (Chanalaris et al., 2003). SAG can bind to metal ions, zinc or copper, and thereby protect cells from metal ion-induced apoptosis (Duan et al., 1999; Swaroop et al., 1999) SAG overexpressed in U937 cells prevents ionizing radiationinduced apoptosis through inhibiting ROS production (Kim et al., 2011). It has been suggested that SAG can attenuate ischemia-induced brain injury in vivo mouse models (Yang et al., 2001) and in gerbil ischemia/reperfusion models (Chanalaris et al., 2003; Kim et al., 2010; Yoo et al., 2012). In addition, SAG overexpression promotes cell growth under serum starvation by mediating p27 ubiquitination and degradation (Duan et al., 2001). Elevated levels of SAG have been found in human colon carcinomas and transfection of antisense SAG into the tumor cells reduced growth of these cells (Huang et al., 2001).

Although many peptides and proteins have remarkable therapeutic potential, their low permeability and poor bioavailability have been obstacles in the process of developing them as drugs. Several useful strategies have been suggested to improve the delivery of proteins into various cells or tissues (Schwarze et al., 1999; van den Berg and Dowdy, 2011). Particularly, protein transduction domains (PTD) including Tat and PEP-1 have the ability to efficiently cross the blood brain barrier (BBB) and cellular membranes. Therefore, exogenous proteins 
can be efficiently delivered to viable tissues and cells through fusion with PTD (Ahn et al., 2010; Lee et al., 2012; Schwarze et al., 1999; van den Berg and Dowdy, 2011).

In this study, we investigated whether Tat-SAG fusion protein could be effectively delivered to SH-SY5Y neuroblastoma cells and brain tissues and so prevent 1-methyl-4-phenylpyridinium $\left(\mathrm{MPP}^{+}\right)$-induced apoptosis in SH-SY5Y cells, and protect DA neurons in the SN in a 1-methyl-4-phenyl-1,2,3,6-tetrahydropyridine (MPTP)-induced PD model. Therefore, we suggest that Tat-SAG may be a useful agent for treatment of ROS-related diseases including PD.

\section{MATERIALS AND METHODS}

\section{Materials and cell culture}

Restriction endonuclease and T4 DNA ligase were purchased from Promega (USA). Plasmid pET-15b and Escherichia coli strain BL21 (DE3) were obtained from Novagen (Germany). Oligonucleotides were synthesized from Bioneer (Korea). An $\mathrm{Ni}^{2+}$-nitrilotriacetic acid Sepharose Superflow column was purchased from Qiagen (USA). Fetal bovine serum (FBS) and antibiotics were purchased from Gibco BRL (USA). MPTP was dissolved in saline and intraperitoneally (i.p.) injected. All other chemicals and reagents, unless otherwise stated, were obtained from Sigma-Aldrich (USA) and were of the highest anaIytical grade available.

\section{Animals}

Male C57BL/6 mice (8-week-old, Hallym University Experimental Animal Center, Korea) were used. They were housed at an ambient temperature of $23^{\circ} \mathrm{C}$ under a $12 \mathrm{~h}$ light $/ 12 \mathrm{~h}$ dark cycle and were provided with food and water ad libitum. All experimental procedures involving animals and their care conformed to the Guide for the Care and Use of Laboratory Animals of the National Veterinary Research \& Quarantine Service of Korea and were approved by the Hallym Medical Center Institutional Animal Care and Use Committee.

\section{Preparation of Tat-SAG and SAG proteins and their transduction into SH-SY5Y cells}

Expression vectors of Tat-SAG and SAG were constructed and two proteins were overexpressed and purified as described previously (Kim et al., 2010). To assess the dose- and timedependency of SAG and Tat-SAG transduction into SH-SY5Y cells, cells were plated into 6-well plates and were cultured in EMEM for $24 \mathrm{~h}$. Cells were incubated with SAG and Tat-SAG in serum-free medium for $60 \mathrm{~min}$. Then cells were harvested by trypsinization for $10 \mathrm{~min}$ and washed with phosphate-buffered saline (PBS) three times. The cells were lysed with a lysis buffer and Western blot analysis was performed. To evaluate the intracellular stability of Tat-SAG, cells were exposed to TatSAG $(3 \mu \mathrm{M})$ for $60 \mathrm{~min}$, and harvested at designated times (1$60 \mathrm{~h})$. The levels of Tat-SAG were analyzed by Western blot analysis using an His antibody and a horseradish peroxidaseconjugated secondary antibody.

\section{Cell viability measurement}

An MTT assay was performed as described previously (Kim et al., 2012). SH-SY5Y cells were seeded into 96 well plates at the density of 10,000 cells/well overnight. The cells were treated with SAG or Tat-SAG in serum-free EMEM medium $1 \mathrm{~h}$ prior to incubation with $\mathrm{MPP}^{+}(4.0 \mathrm{mM})$. After $18 \mathrm{~h}$ incubation, MTT was added to each well for $4 \mathrm{~h}$ and the formazan crystal was dissolved in DMSO. Optical density was measured at $590 \mathrm{~nm}$ using an ELISA plate reader.

\section{ROS generation}

ROS generation was assessed by determining fluorescence intensity derived from a process converting 2',7'-dichlorofluorescein diacetate (DCF-DA) into fluorescent DCF. SH-SY5Y cells were plated into 96 well plates and pretreated with or without SAG and Tat-SAG for $60 \mathrm{~min}$. This was followed by incubation with $\mathrm{MPP}^{+}(4.0 \mathrm{mM})$ for 30 min and staining with DCF-DA $(20 \mu \mathrm{M})$ for $30 \mathrm{~min}$. The fluorescence was detected using a fluorescence microscope (Nikon, Japan) at $485 \mathrm{~nm}$ excitation and $538 \mathrm{~nm}$ emission.

\section{Western blot analysis}

Tissue samples were homogenized using a microtube homogenizer and cells were lysed in a lysis buffer. The protein concentrations in cell lysates or tissue homogenates were determined using a protein assay kit. Equal amounts of protein samples were resolved by $15 \%$ sodium dodecyl sulfate-polyacrylamide gel electrophoresis (SDS-PAGE) and then electrotransferred to a nitrocellulose membrane. The membrane was blocked with $5 \%$ non-fat dry milk in TBS-T buffer $(25 \mathrm{mM}$ Tris$\mathrm{HCl}, 140 \mathrm{mM} \mathrm{NaCl}, 0.1 \%$ Tween 20, $\mathrm{pH} 7.5$ ) and was incubated with a primary and secondary antibody. The membrane was developed with enhanced chemiluminescence reagents according to the manufacturer's instructions (USA). The $\beta$-actin level in each sample was used as a loading control. The band densities were analyzed by image analysis software.

\section{Confocal fluorescence microscopy}

After transduction of SAG and Tat-SAG into SH-SY5Y cells, their intracellular distributions were assessed by confocal fluorescence microscopy. Cells were grown on glass coverslips and exposed to SAG and Tat-SAG $(3 \mu \mathrm{M})$ for $60 \mathrm{~min}$. The cells were washed with PBS three times and fixed with $4 \%$ paraformaldehyde at room temperature for $10 \mathrm{~min}$, followed by incubation with an anti-histidine antibody and Alexa fluor 488-conjugated secondary antibody (Invitrogen, USA). 4'6-diamidino-2phenylindole (DAPI) was used for nuclear counterstaining. The fluorescence image was analyzed using an Olympus FV-300 confocal fluorescence microscope.

\section{Terminal deoxynucleotidyl transferase-mediated dUTP nick end labeling (TUNEL) assay}

To assess the effects of SAG or Tat-SAG on MPP ${ }^{+}$-induced DNA fragmentation, SH-SY5Y cells were pre-incubated with Tat, SAG, and Tat-SAG for $1 \mathrm{~h}$, followed by treatment with $\mathrm{MPP}^{+}(4.0 \mathrm{mM})$ for an additional $18 \mathrm{~h}$. TUNEL staining was performed using a Cell Death Detection kit (Roche Applied Science, Switzerland) according to the manufacturer's instructions.

\section{In vivo transduction of Tat fusion protein}

To assess whether SAG and Tat-SAG proteins would traverse the BBB, SAG and Tat-SAG (3 mg/kg body weight) were i.p. injected into the mice ( $n=7$ mice/group). The mice were sacrificed $12 \mathrm{~h}$ later. The distribution of SAG and Tat-SAG in the SN were evaluated by histological analysis using a rabbit antihistidine polyclonal antibody and biotinylated goat anti-rabbit secondary antibody.

Protein treatment in MPTP-induced PD animal model Mice were divided into 5 groups as follows; control, MPTP, MPTP + SAG, MPTP + Tat, and MPTP + Tat-SAG ( $\mathrm{n}=7 \mathrm{mice} /$ group). Saline, SAG, Tat, and Tat-SAG ( $3 \mathrm{mg} / \mathrm{kg}$ body weight) 
A

\begin{tabular}{rl|l|} 
Tat-SAG & His - Tat & SAG \\
SAG & His \\
Tat & Tat & \\
& &
\end{tabular}

B

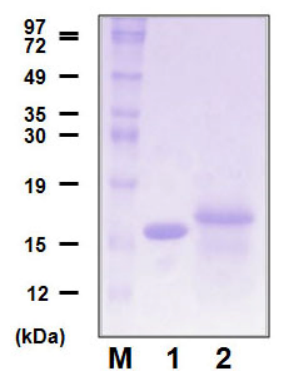

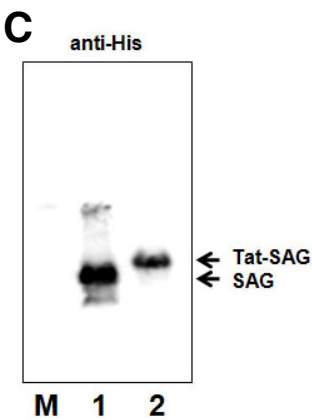

$\begin{array}{lll}\text { M } 1 & 2\end{array}$

Fig. 1. Overexpression and purification of Tat-SAG protein. (A) Schematic structure of SAG and Tat-SAG. The His sequence was used for purification and detection of both SAG and Tat-SAG in Western blot analysis. (B) SDS-PAGE and (C) Western blot analysis of purified SAG and Tat-SAG. Overexpressed SAG and Tat$\mathrm{SAG}$ proteins were purified using $\mathrm{Ni}^{2+}$-nitrilotriacetic acid Sepharose column. The purified SAG and Tat-SAG were analyzed by $15 \%$ SDS-PAGE, electrotransferred to nitrocellulose membrane, and probed with an anti-His and secondary antibody. Lane M, molecular weight marker (EBM-1035, Elpisbiotech, Korea); lane 1, SAG; lane 2, Tat-SAG.

were i.p. administered to mice and then MPTP $(20 \mathrm{mg} / \mathrm{kg}$ body weight) were injected four times at $2 \mathrm{~h}$ intervals the following day. The mice were sacrificed 7 days after MPTP treatment. Animals were anesthestized by an i.p. injection with sodium pentobarbital (100 mg/kg body weight) and fixed transcardially with $4 \%$ paraformaldehyde. The brains were post-fixed in the same fixative for $4 \mathrm{~h}$, frozen and sectioned. Staining of tissue sections was carried out as previously described (Kim et al., 2008). Sections were blocked by $3 \%$ bovine serum albumin in PBS for $30 \mathrm{~min}$ and were probed with primary antibodies [His and tyrosine hydroxylase (TH)] and a biotinylated secondary antibody. Also, cresyl violet staining was performed to detect viable cells after $\mathrm{TH}$ immunostaining. The sections were visualized with 3,3'-diaminobenzidine (DAB) in $0.1 \mathrm{M}$ Tris buffer and mounted on gelatin-coated slide. Images were captured and analyzed using an Olympus DP72 digital camera. The sections were processed and analyzed by an observer who was blinded with regard to mouse groups.

\section{Behavior test}

To assess the motor functions of mice, a Rotarod test was performed using Rotarod, Series 8 (IITC, USA) as described previously (Jeong et al., 2012). The acceleration of rotation gradually increased from 0 to $45 \mathrm{rpm}$. Each mouse was placed on the rotarod. The distance $(\mathrm{m})$ and time $(\mathrm{s})$ that each mouse spent on the rotarod without falling were automatically recorded.

\section{Statistics}

Data are expressed as the means \pm SD. Comparison between groups was performed by Student's $t$-test. Values of $P<0.05$, $P<0.01, P<0.005$, and $P<0.001$ were considered to be statistically significant.
A

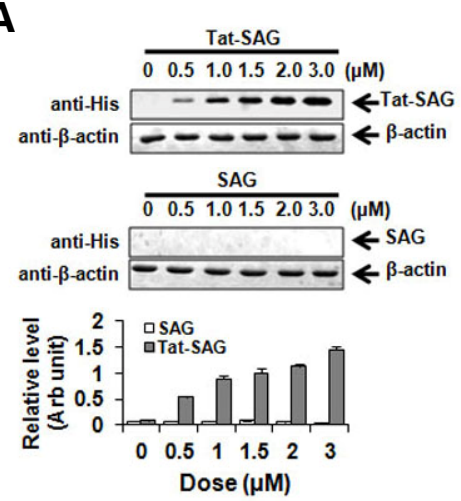

C

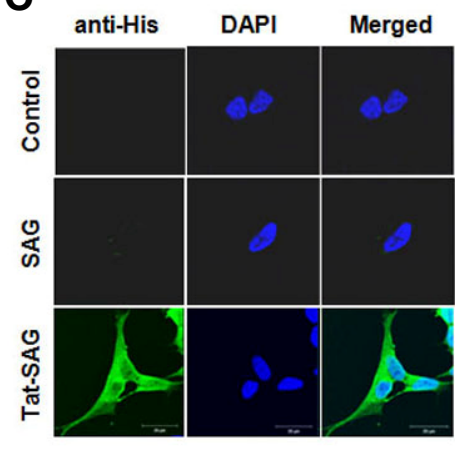

B
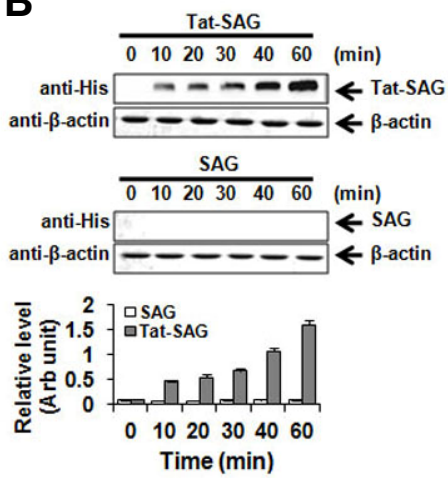

D

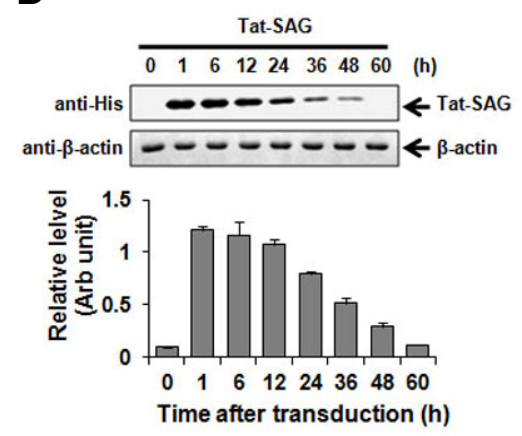

Fig. 2. In vitro transduction of Tat-SAG into SHSY5Y cells. (A) To assess the dose-dependency of transduction of SAG and Tat-SAG, SH-SY5Y cells were incubated with various concentrations of $S A G$ and Tat-SAG (0.5-3.0 $\mu \mathrm{M})$ for $60 \mathrm{~min}$. (B) To assess a time-dependency of transduction of SAG and TatSAG, SH-SY5Y cells were incubated with SAG and Tat-SAG $(3.0 \mu \mathrm{M})$ for various time periods $(10-60$ min). The cells were treated with EDTA-trypsin for 10 min, washed with PBS three times, and harvested by centrifugation. Transduced SAG and TatSAG in cell extracts were identified by SDS-PAGE and Western blot analysis using a histidine and a horseradish peroxidase-conjugated secondary antibody. Cellular level of each protein was quantified using a densitometer and bar graph represents the $\beta$-actin normalized data. (C) Fluorescence microscopic data showing a transduction of SAG and TatSAG into SH-SY5Y cells. Cells were exposed to SAG and Tat-SAG $(3 \mu \mathrm{M})$ for $60 \mathrm{~min}$ and two proteins were probed with a rabbit anti-histidine and Alexa fluor 488-conjugated anti-rabbit secondary antibody. (D) Intracellular stability of transduced TatSAG. Purified Tat-SAG $(3 \mu \mathrm{M})$ was transduced into cells for $60 \mathrm{~min}$ and the intracellular level of exogenous Tat-SAG was measured by Western blot analysis for $60 \mathrm{~h}$. The bar graph represents means \pm SD from three independent experiments. Arb. unit, arbitrary unit. 
A
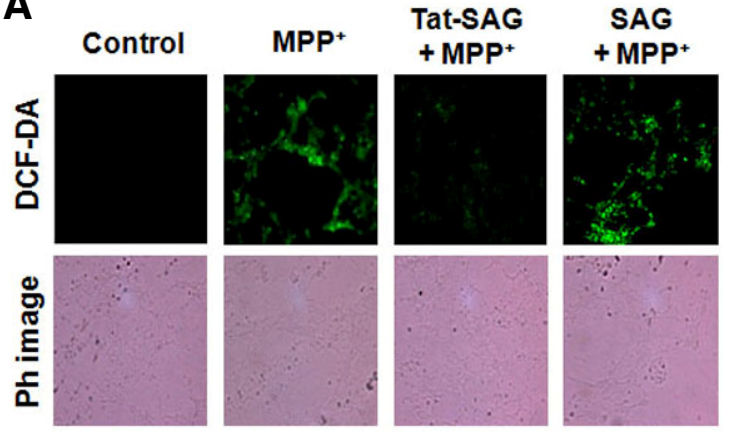

Tat $+\mathrm{MPP}^{+}$
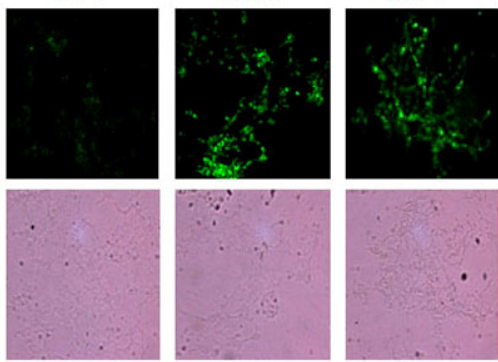

B

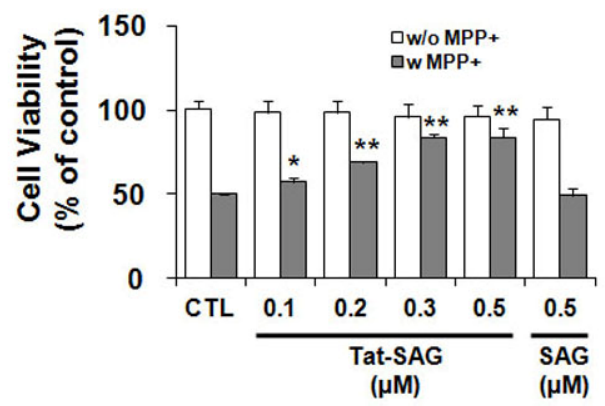

Fig. 3. Effect of Tat-SAG on MPP ${ }^{+}$-induced ROS generation and cell death. (A) Effect of SAG and Tat-SAG on $\mathrm{MPP}^{+}$-induced ROS generation in SH-SY5Y cells. SAG and Tat-SAG were transduced into SH-SY5Y cells prior to $\mathrm{MPP}^{+}$ treatment $(4.0 \mathrm{mM})$ for $30 \mathrm{~min}$. The cells were exposed to a fluorescent dye, DCF-DA, and then observed by fluorescent microscopy. Ph image, Phase contrast image. (B) Effects of SAG and Tat-SAG on MPP ${ }^{+}$-induced toxicity in SH-SY5Y cells. Cells were preincubated with SAG and Tat-SAG (0.1$0.5 \mu \mathrm{M})$ and cellular toxicity was induced by $\mathrm{MPP}^{+}(4.0 \mathrm{mM})$ for $18 \mathrm{~h}$. Cell viability was assessed using an MTT assay. Effects of SAG and Tat-SAG on SH-SY5Y cell survival were compared in the presence or absence of $\mathrm{MPP}^{+}$. The bar graph represents means \pm SD from three independent experiments. Data were analyzed by Student's $t$-test. ${ }^{*} P<$ 0.05 and ${ }^{* *} P<0.01$ indicate a statistically significant difference between $\mathrm{MPP}^{+}$-treated control and Tat-SAG-treated samples.

\section{RESULTS}

\section{Tat-SAG transduced into SH-SY5Y cells in a time- and dose-dependent manner}

Expression vectors of SAG and Tat-SAG, a SAG protein fused with a Tat peptide, were constructed and their schematic structures were shown in Fig. 1A. Two proteins were purified as described previously (Kim et al., 2010) and their purities were confirmed by SDS-PAGE and Western blot analysis (Figs. 1B and $1 \mathrm{C})$.

We first examined the abilities of SAG and Tat-SAG to penetrate into SH-SY5Y cells. SH-SY5Y cells were exposed to SAG and Tat-SAG $(0.5-3.0 \mu \mathrm{M})$ for $60 \mathrm{~min}$ and the transduced protein levels in the cells were measured by Western blot analysis using an anti-His antibody. As shown in Fig. 2A, Tat-SAG levels increased in a time-dependent manner, compared to the level of SAG, which was not detected in the cells. In addition, TatSAG transduced into the cells in a time-dependent manner as observed when treated with Tat-SAG $(3.0 \mu \mathrm{M})$ for various incubation times (10-60 min) (Fig. 2B). Immunofluorescence data revealed that Tat-SAG was significantly detected in the nucleus as well as cytoplasm of SH-SY5Y cells (Fig. 2C). We next evaluated the stability of Tat-SAG protein after its transduction into SH-SY5Y cells. Western blot analysis showed that the Tat-SAG level at $48 \mathrm{~h}$ was reduced to $24 \%$ of the initial level $(1 \mathrm{~h}$ after transduction) (Fig. 2D). A significant level of Tat-SAG was observed in SH-SY5Y cells for 1-60 $\mathrm{h}$ after its transduction. These results suggest that Tat-SAG effectively transduced into $\mathrm{SH}$ SY5Y cells and further, that the intracellular Tat-SAG level was significantly maintained for $48 \mathrm{~h}$.

\section{Transduced Tat-SAG inhibits ROS production and cell death}

SAG is known to scavenge ROS induced by redox agents (Duan et al., 1999). To confirm whether Tat-SAG would inhibit ROS generation, SH-SY5Y cells were treated with $\mathrm{MPP}^{+}(4.0$ $\mathrm{mM}) 30$ min after exposure to SAG, Tat, or Tat-SAG for $60 \mathrm{~min}$.
SAG and Tat failed to suppress ROS generation caused by $\mathrm{MPP}^{+}$. In contrast, an obvious decrease in the fluorescent signal was observed in the MPP ${ }^{+}+$Tat-SAG treated sample (Fig. 3A). Next we assessed whether Tat-SAG could inhibit MPP ${ }^{+}$induced toxicity in SH-SY5Y cells using an MTT assay. The MTT assay showed that, when treated only with SAG or TatSAG, neither SAG nor Tat-SAG affected the proliferation of SHSY5Y cells. However, when treated with MPP', Tat-SAG (0.1$0.5 \mu \mathrm{M})$ significantly suppressed $\mathrm{MPP}^{+}$-induced toxicity in a dose-dependent manner (Fig. 3B). These results demonstrate that Tat-SAG significantly inhibited ROS production in response to $\mathrm{MPP}^{+}$and this was associated with suppression of $\mathrm{MPP}^{+}$induced toxicity.

Tat-SAG protects SH-SY5Y neuroblastoma cells by inhibiting DNA fragmentation and apoptosis induced by MPP ${ }^{+}$ SAG overexpression is known to protect cells from apoptosis through scavenging ROS and inhibiting caspase activation (Duan et al., 1999). To examine whether Tat-SAG suppresses DNA fragmentation caused by exposure to $\mathrm{MPP}^{+}$, a TUNEL assay was carried out after SH-SY5Y cells were treated with MPP $^{+}$following transduction of SAG and Tat-SAG for $1 \mathrm{~h}$. Transduction of Tat-SAG remarkably decreased the number of TUNEL labeled cells in contrast with the SAG and Tat treated samples (Fig. 4A). The percentage of fluorescently labeled cells in the Tat-SAG-treated sample was reduced to approximately $55 \%$, compared with the $\mathrm{MPP}^{+}$-treated sample (Fig. 4B). Further, we investigated whether Tat-SAG could affect MPP ${ }^{+}$triggered apoptosis progression in SH-SY5Y cells. Tat-SAG markedly suppressed elevated levels of cleaved caspase-3 and bax, apoptotic markers, and also significantly up-regulated reduced levels of bcl-2, an anti-apoptotic marker (Figs. 4C and 4D). These findings demonstrate that transduced Tat-SAG inhibits $\mathrm{MPP}^{+}$-induced DNA fragmentation and apoptosis in $\mathrm{SH}-$ SY5Y cells, possibly by protecting $\mathrm{SH}-\mathrm{SY} 5 \mathrm{Y}$ neuroblastoma cells from oxidative stress caused by MPP ${ }^{+}$. 
A

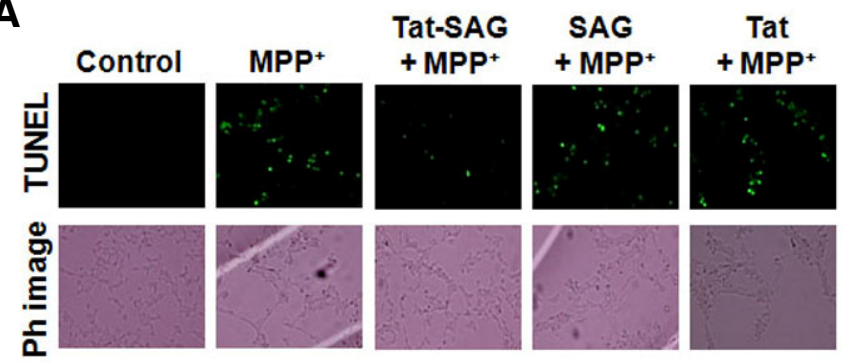

B

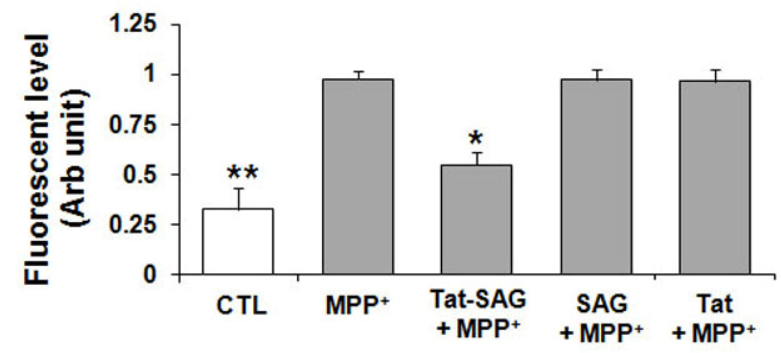

C

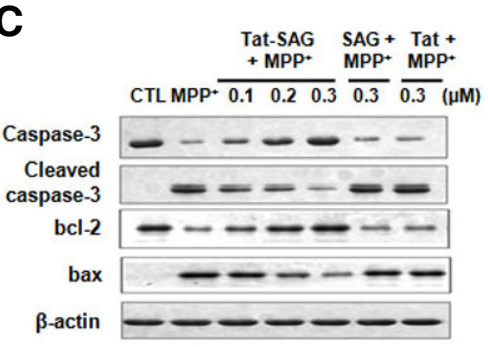

D $\bar{\Phi} 1.5 \quad$ aCas-3
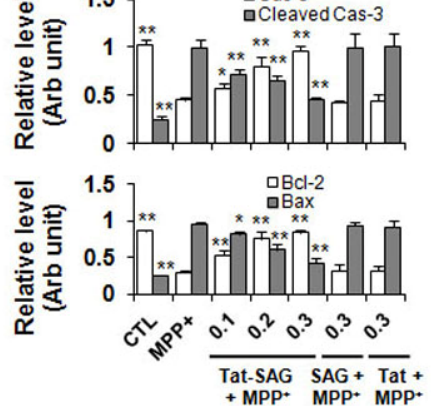

Fig. 4. Tat-SAG inhibits $M P P^{+}$-induced apoptosis in SH-SY5Y cells. SH-SY5Y cells were incubated with Tat, SAG, or Tat-SAG $(0.3 \mu \mathrm{M})$ for $60 \mathrm{~min}$, followed by treatment with $\mathrm{MPP}^{+}(4.0 \mathrm{mM})$ for additional $18 \mathrm{~h}$. (A) A level of damaged DNA and (B) a fluorescent level of each sample was quantified by TUNEL assay. ${ }^{*} P<$ 0.05 and ${ }^{* *} P<0.01$ for $\mathrm{MPP}^{+}$versus other groups. (C, D) Effect of Tat-SAG on the levels of pro- or antiapoptotic markers. Levels of caspase-3, cleaved caspase-3, Bcl-2, and Bax were evaluated using (C) Western blot analysis and (D) were quantified using a densitometer. The following antibodies were used; mouse anti-caspase-3, mouse anti-cleaved caspase-3, mouse anti-bcl-2, mouse anti-bax, and mouse anti- $\beta$-actin. Western blotting analysis was independently repeated three times. Data were analyzed by Student's $t$-test. ${ }^{*} P$ $<0.05$ and ${ }^{\star \star} P<0.001$ indicate a statistically significant difference between $\mathrm{MPP}^{+}$-treated group and other groups.
Transduced Tat-SAG protects dopaminergic neurons in the SN from MPTP, a neurotoxin

We next evaluated the ability of Tat-SAG to efficiently cross the BBB as well as to enter SH-SY5Y cells. SAG and Tat-SAG were i.p. injected into mice ( $3 \mathrm{mg} / \mathrm{kg}$ body weight, $\mathrm{n}=7 \mathrm{mice} /$ group) and mice brains were harvested $12 \mathrm{~h}$ later. Brain tissues were sectioned and stained using anti-histidine and biotinylated secondary antibodies. As shown in Fig. 5A, histological analysis showed that transduced Tat-SAG proteins were found in the, predominantly, cytoplasm as well as nucleus of DA neurons in the brain parenchyma, indicating that Tat-SAG has the capability to cross the BBB, in contrast with SAG.

It has been shown that MPTP is converted to $\mathrm{MPP}^{+}$in glial cells and subsequently MPP ${ }^{+}$induces the death of DA neurons in the SN. We examined whether Tat-SAG can protect DA neurons in the SN against MPTP toxicity in a MPTP-induced PD mouse model. Mice ( $n=7$ mice/group) were i.p. injected with SAG and Tat-SAG ( $3 \mathrm{mg} / \mathrm{kg}$ body weight) and the next day MPTP ( $20 \mathrm{mg} / \mathrm{kg}$ body weight) was i.p. injected 4 times. The mice in the Tat-SAG group had significantly more $\mathrm{TH}$ positive cells in the SN, although less than the control mice, when compared to the MPTP-treated sample (Left panels, Fig. 5B). Furthermore, to confirm the survival of DA neurons in the SN of the mice after MPTP treatment, brain tissues were double-stained using a TH antibody and cresyl violet. Cresyl violet staining showed that MPTP treatment significantly induced the death of
DA neurons in the SN. As expected, a significant number of viable TH neurons were seen in the Tat-SAG-treated sample (Right panels, Fig. 5B). The quantification data of TH positive cells shows that Tat-SAG protects TH positive neurons from MPTP (Fig. 5C). Next, to assess the effect of Tat-SAG on the motor functions in the MPTP-treated mice, we measured the distance and time that mice could stay on a moving rotarod without falling. The Tat-SAG-treated group spent significantly more time and moved longer distances on a rod, although less and shorter than the control group, compared to the MPTP, SAG, and Tat treated groups (Figs. 5D and 5E). Therefore, the results demonstrate that Tat-SAG represses the death of DA neurons in the SN caused by MPTP, thereby leading to a decrease in impairment of motor functions.

\section{DISCUSSION}

Neurodegenerative diseases, including Alzheimer's disease, Huntington's disease, and Parkinson's disease, are different from each other in terms of their causes and symptoms as well as the affected area of the brain (Chinta and Andersen, 2008; Dawson and Dawson, 2003; Miller et al., 2009; Ramalingam and Kim, 2012). However, excessive ROS generation seems to be tightly related to these neurodegenerative diseases. Mitochondria produce ROS and also neutralize ROS via the functions of various defense systems, which they possess, such as 
A

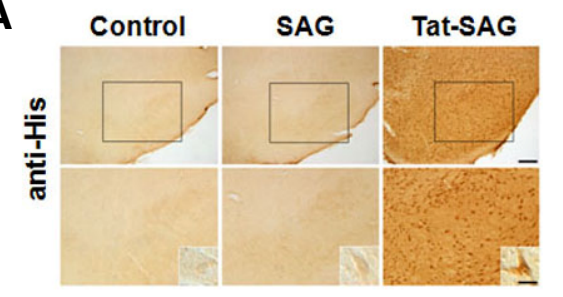

B
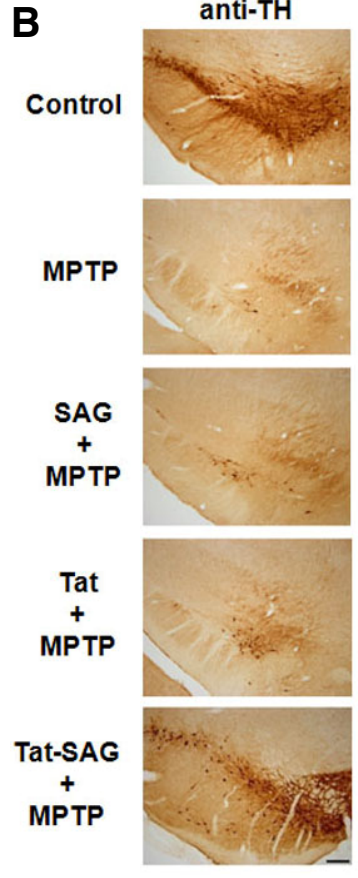

anti-TH + CV

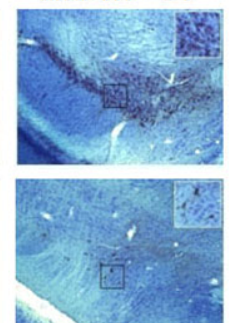

D

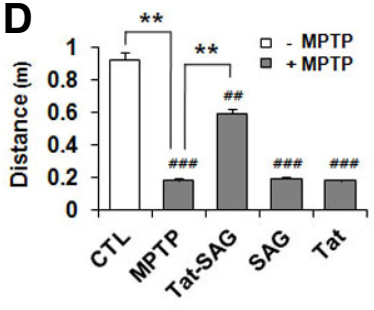

E

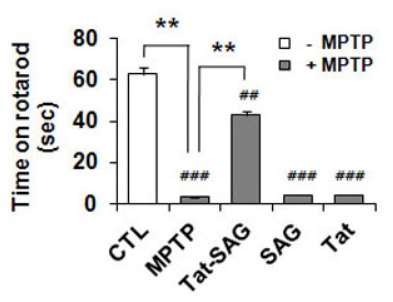

Fig. 5. Tat-SAG protects DA neurons in the SN against MPTP toxicity in the MPTP-induced PD model. (A) Photomicrographs showing transduction of TatSAG into the SN. The brains were taken at $12 \mathrm{~h}$ after injection of SAG and Tat-SAG at a dose of $3 \mathrm{mg} / \mathrm{kg}$ body weight per mouse ( $\mathrm{n}=7$ mice/group) and immunostained with a rabbit anti-histidine antibody and biotinylated goat anti-rabbit secondary antibody. Lower panels, images with high magnification. (B) Protective effect of Tat-SAG on MPTP-induced cell death of DA neurons in the SN. Left panel, stained with an anti-TH antibody against DA neurons; right panel, double-stained with a TH antibody and cresyl violet for Nissl bodies. The shown images are representatives from each group. Scale bars, $100 \mu \mathrm{m}$ for $(\mathrm{A}, \mathrm{B}) ; 50 \mu \mathrm{m}$ for high magnifications in (A, B). (C) The number of TH positive DA neurons in the SN. Quantification of the number of DA neurons in $250 \times$ $250 \mu \mathrm{m}^{2}$ ( $\mathrm{n}=7 \mathrm{mice} / \mathrm{group}$ ) is shown in the graph. (D-E) Effect of Tat-SAG on the behavior of mice in a MPTP-induced PD model. Each mouse was placed on a Rotarod. (D) The distance and (E) the time that the mice stayed on a Rotarod without falling were recorded. All groups are as follows; CTL, untreated control; MPTP, MPTP-treated group; Tat-SAG, TatSAG and MPTP-treated group; SAG, SAG and MPTP-treated group; Tat, Tat and MPTP-treated group. ${ }^{* *} P<0.001$ for MPTP versus other groups; ${ }^{\#} P<0.05,{ }^{\# \#} P<0.005$, and ${ }^{\# \# \#} P<0.001$ for control versus other groups. glutathione, glutathione peroxidase, superoxide dismutase, and NADPH (Ramalingam and Kim, 2012). Therefore, it has been suggested that, if antioxidant molecules are up-regulated in target tissues or cells susceptible to oxidative damage, they may prevent oxidative stress and subsequent cell death. We produced PTD-fused proteins, including PEP-1-rpS3 and TatANX, using PTD tools in order to increase therapeutic proteins and peptides in any cell type and tissues. These proteins, including PEP-1-rpS3 and Tat-ANX, can be efficiently delivered to cells and animal tissues, and they can inhibit ROS-related diseases involving inflammation and ischemic insult in animal models (Ahn et al., 2010; Lee et al., 2012).

$\mathrm{SAG}$, also known as RBX2/ROC2, a protein that is a component of the Skp1-Cullin1-F-box-protein E3 ubiqutin ligase (Tan et al., 2006b; Wei and Sun, 2010), was identified as a redoxinducible antioxidant protein (Duan et al., 1999; Swaroop et al., 1999) and is induced by various stimuli, including redox agents, ischemia/reoxygenation, hypoxia, neurotoxin, heat shock, and UV irradiation (Wei and Sun, 2010). Human SAG protein possesses the binding sites for a lot of transcription factors, such as NF- $\mathrm{KB}, \mathrm{c}-\mathrm{MYC}, \mathrm{AP}-1$ and $\mathrm{p53}$, and also it can bind to zinc and copper metal ions, which can induce apoptosis (Duan et al., 1999; Jia et al., 2010). Numerous studies have shown that SAG functions as an antioxidant or anti-apoptotic protein. For example, SAG knockdown in human prostate cancer PC3 using siRNA, increased the susceptibility to inducers of ROS pro- duction or apoptosis, such as actinomycin D and etoposide (Yang et al., 2010). It is known that in multiple human tumor tissues, overexpression of SAG lead to reduced levels and half life of NOXA, a pro-apoptotic member of the Bcl-2 family. Also, SAG knockdown in cancer cells could sensitize the cells to radiation and induce apoptosis through accumulation of NOXA (Jia et al., 2010). SAG-overexpressing SH-SY5Y cells inhibited $\mathrm{MPP}^{+}$-induced neurotoxicity by down-regulation of ROS generation and c-Jun N-terminal kinase 1 activity (Kim et al., 2007). SAG overexpression in mouse epidermal cells promotes ultraviolet B-induced skin hyperplasia, but not skin tumors (He et al., 2008). In addition, SAG suppresses TPA-induced neoplastic transformation via ubiquitination and degradation of c-Jun (Gu et al., 2007) and protects cells from apoptosis by promoting the ubiquitination and degradation of procaspase-3 (Tan et al., 2006a). Thus, these reports suggested that an increased level of SAG could enhance survival of various cells.

As is well-known, in the intrinsic apoptotic pathway various apoptosis inducers, including ROS, induce the loss of mitochondrial membrane potential and release of pro-apoptotic proteins, such as Bax, into the cytosol. Consequently, it leads to activation of caspases and DNA fragmentation, a hallmarker of apoptosis. Consistent with other previous studies, we demonstrated that Tat-SAG significantly suppresses signaling of apoptosis including ROS generation (Fig. 3A), release of Bax, and activation of caspase-3 (Figs. 4C and 4D), thereby leading to sup- 
pression of DNA fragmentation (Figs. $4 \mathrm{~A}$ and $4 \mathrm{~B}$ ) and cell death (Fig. 3B). Chanalaris et al. reported that SAG is found in both the cytoplasm and nucleus of rat cardiomyocytes (Chanalaris et al., 2003). Their findings were consistent with our results which showed that Tat-SAG was localized in the cytoplasm and nucleus of SH-SY5Y cells, although different from the cell line used in their studies. However, until now it was not evident that either Tat-SAG in the cytoplasm or nucleus can predominantly prevent $\mathrm{MPP}^{+}$-induced apoptosis or otherwise what role the transduced Tat-SAG can play in the nucleus of SH-SY5Y cells. Therefore, additional studies were required to clearly reveal the function of Tat-SAG in the SH-SY5Y cells.

Furthermore, our in vivo study revealed that Tat-SAG has the capability to cross the BBB and, through just a single administration of Tat-SAG, protects DA neurons in the SN against MPTP toxicity, subsequently maintaining the motor functions in the Tat-SAG-treated mice (Fig. 5). In this regard, we clearly showed that the increase of cellular levels of SAG enhances the cellular viability of the SH-SY5Y neuroblastoma cell line and DA neurons in the SN. Also, we previously reported that TatSAG significantly protects astrocyte cells and the CA1 region in the hippocampus of gerbil brains from ischemic damage (Kim et al., 2010).

The BBB is known as an obstacle to the delivery of therapeutic molecules to brain. It is therefore noteworthy that Tat-SAG, which has the capability to reduce oxidative stress-induced toxicity, could be effectively and easily delivered to cells and brain tissues, as shown in several studies including our data. On the other hand, we identified that PTD fusion proteins, including PEP-1-rpS3 and Tat-frataxin, are retained in cells and tissues for a considerable period and then gradually disappear (unpublished data). These results seem to demonstrate another advantage of PTD fusion proteins. Continuous overexpression in some cells and tissues could render excessive growthpromoting conditions to normal cells and tissues, thereby likely causing side effects. Consequently, our present study is considered as another promising observation showing that PTDfusion proteins could provide appropriate therapeutic agents against diseases such as PD.

In conclusion, the present study demonstrates that Tat-SAG can cross cellular membranes and the BBB, and suppress ROS generation, DNA damage, and cell death induced by oxidative stress and consequently protect DA neurons in the SN in a PD animal model. Taken collectively, it is likely that Tat-SAG could be a promising agent against ROS-related diseases such as inflammation and PD.

\section{ACKNOWLEDGMENTS}

This work was supported by a Priority Research Centers Program grant (NRF-2009-0093812) and a Basic Science Research grant (2012R1A1A2006995) through the National Research Foundation of Korea funded by the Ministry of Science, ICT and Future Planning and in part by a grant (HI12C086 90200) of the Korean Health Technology R\&D Project, Ministry of Health \& Welfare, Republic of Korea.

\section{REFERENCES}

Ahn, E.H., Kim, D.W., Kang, H.W., Shin, M.J., Won, M.H., Kim, J., Kim, D.J., Kwon, O.S., Kang, T.C., Han, K.H., et al. (2010). Transduced PEP-1-ribosomal protein S3 (rpS3) ameliorates 12-Otetradecanoylphorbol-13-acetate-induced inflammation in mice. Toxicology 276, 192-197.

Bae, Y.S., Oh, H., Rhee, S.G., and Yoo, Y.D. (2011). Regulation of reactive oxygen species generation in cell signaling. Mol. Cells 32, 491-509.
Chanalaris, A., Sun, Y., Latchman, D.S., and Stephanou, A. (2003). SAG attenuates apoptotic cell death caused by simulated ischaemia/reoxygenation in rat cardiomyocytes. J. Mol. Cell. Cardiol. 35, 257-264.

Chinta, S.J., and Andersen, J.K. (2008). Redox imbalance in Parkinson's disease. Biochim. Biophys. Acta 1780, 1362-1367.

Dawson, T.M., and Dawson, V.L. (2003). Molecular pathways of neurodegeneration in Parkinson's disease. Science 302, 819-822.

Duan, H., Wang, Y., Aviram, M., Swaroop, M., Loo, J.A., Bian, J., Tian, Y., Mueller, T., Bisgaier, C.L., and Sun, Y. (1999). SAG, a novel zinc RING finger protein that protects cells from apoptosis induced by redox agents. Mol. Cell. Biol. 19, 3145-3155.

Duan, H., Tsvetkov, L.M., Liu, Y., Song, Y., Swaroop, M., Wen, R., Kung, H.F., Zhang, H., and Sun, Y. (2001). Promotion of Sphase entry and cell growth under serum starvation by SAG/ ROC2/Rbx2/Hrt2, an E3 ubiquitin ligase component: association with inhibition of p27 accumulation. Mol. Carcinog. 30, 37-46.

Gu, Q., Tan, M., and Sun, Y. (2007). SAG/ROC2/Rbx2 is a novel activator protein-1 target that promotes c-Jun degradation and inhibits 12-O-tetradecanoylphorbol-13-acetate-induced neoplastic transformation. Cancer Res. 67, 3616-3625.

He, H., Gu, Q., Zheng, M., Normolle, D., and Sun, Y. (2008). SAG/ ROC2/RBX2 E3 ligase promotes UVB-induced skin hyperplasia but not skin tumors, by simultaneously targeting C-Jun/AP-1 and p27. Carcinogenesis 29, 858-865.

Huang, Y., Duan, H., and Sun, Y. (2001). Elevated expression of SAG/ROC2/Rbx2/Hrt2 in human colon carcinomas: SAG does not induce neoplastic transformation, but antisense SAG transfection inhibits tumor cell growth. Mol. Carcinog. 30, 62-70.

Jeong, H.J., Kim, D.W., Woo, S.J., Kim, H.R., Kim, S.M., Jo, H.S., Park, M., Kim, D.S., Kwon, O.S., Hwang, I.K., et al. (2012). Transduced Tat-DJ-1 protein protects against oxidative stressinduced SH-SY5Y cell death and Parkinson disease in a mouse model. Mol. Cells 33, 471-478.

Jia, L., Yang, J., Hao, X., Zheng, M., He, H., Xiong, X., Xu, L., and Sun, Y. (2010). Validation of SAG/RBX2/ROC2 E3 ubiquitin ligase as an anticancer and radiosensitizing target. Clin. Cancer Res. 16, 814-824.

Kim, S.Y., Kim, M.Y., Mo, J.S., Park, J.W., and Park, H.S. (2007). SAG protects human neuroblastoma SH-SY5Y cells against 1methyl-4-phenylpyridinium ion (MPP+)-induced cytotoxicity via the downregulation of ROS generation and JNK signaling. Neurosci. Lett. 413, 132-136.

Kim, D.S., Kim, J.E., Kwak, S.E., Choi, K.C., Kim, D.W., Kwon, O.S. Choi, S.Y., and Kang, T.C. (2008). Spatiotemporal characteristics of astroglial death in the rat hippocampo-entorhinal complex following pilocarpine-induced status epilepticus. J. Comp. Neurol. 511, 581-598.

Kim, D.W., Lee, S.H., Jeong, M.S., Sohn, E.J., Kim, M.J., Jeong, H.J., An, J.J., Jang, S.H., Won, M.H., Hwang, I.K., et al. (2010) Transduced Tat-SAG fusion protein protects against oxidative stress and brain ischemic insult. Free Radic. Biol. Med. 48, 969977.

Kim, S.Y., Yang, E.S., Lee, Y.S., Lee, J., and Park, J.W. (2011). Sensitive to apoptosis gene protein regulates ionizing radiationinduced apoptosis. Biochimie 93, 269-276.

Kim, D.S., Sohn, E.J., Kim, D.W., Kim, Y.N., Eom, S.A., Yoon, G.H., Cho, S.W., Lee, S.H., Hwang, H.S., Cho, Y.S., et al. (2012). PEP-1-p18 prevents neuronal cell death by inhibiting oxidative stress and Bax expression. BMB Rep. 45, 532-537.

Lee, S.H., Kim, D.W., Eom, S.A., Jun, S.Y., Park, M., Kim, D.S., Kwon, H.J., Kwon, H.Y., Han, K.H., Park, J., et al. (2012). Suppression of 12-O-tetradecanoylphorbol-13-acetate (TPA)-induced skin inflammation in mice by transduced Tat-Annexin protein. BMB Rep. 45, 354-359.

Miller, R.L., James-Kracke, M., Sun, G.Y., and Sun, A.Y. (2009). Oxidative and inflammatory pathways in Parkinson's disease. Neurochem. Res. 34, 55-65.

Ramalingam, M., and Kim, S.J. (2012). Reactive oxygen/nitrogen species and their functional correlations in neurodegenerative diseases. J. Neural. Transm. 119, 891-910.

Schwarze, S.R., Ho, A., Vocero-Akbani, A., and Dowdy, S.F. (1999) In vivo protein transduction: delivery of a biologically active protein into the mouse. Science 285, 1569-1572.

Swaroop, M., Bian, J., Aviram, M., Duan, H., Bisgaier, C.L., Loo, J.A., and Sun, Y. (1999). Expression, purification, and biochemi- 
cal characterization of SAG, a ring finger redox-sensitive protein. Free Radic. Biol. Med. 27, 193-202.

Tan, M., Gallegos, J.R., Gu, Q., Huang, Y., Li, J., Jin, Y., Lu, H., and Sun, Y. (2006a). SAG/ROC-SCF beta-TrCP E3 ubiquitin ligase promotes pro-caspase-3 degradation as a mechanism of apoptosis protection. Neoplasia 8, 1042-1054.

Tan, M., Zhu, Y., Kovacev, J., Zhao, Y., Pan, Z.Q., Spitz, D.R., and Sun, Y. (2006b). Disruption of Sag/Rbx2/Roc2 induces radiosensitization by increasing ROS levels and blocking NFkappaB activation in mouse embryonic stem cells. Free Radic. Biol. Med. 49, 976-983.

van den Berg, A., and Dowdym, S.F. (2011). Protein transduction domain delivery of therapeutic macromolecules. Curr. Opin. Biotechnol. 22, 888-893.

Wei, D., and Sun, Y. (2010). Small RING finger proteins RBX1 and RBX2 of SCF E3 ubiquitin ligases: the role in cancer and as cancer targets. Genes Cancer 1, 700-707.
Yang, G.Y., Pang, L., Ge, H.L., Tan, M., Ye, W., Liu, X.H., Huang, F.P., Wu, D.C., Che, X.M., Song, Y., et al. (2001). Attenuation of ischemia-induced mouse brain injury by SAG, a redox-inducible antioxidant protein. J. Cereb. Blood Flow Metab. 21, 722-733.

Yang, E.S., Huh, Y.J., and Park, J.W. (2010). Knockdown of sensitive to apoptosis gene by small interfering RNA enhances the sensitivity of PC3 cells toward actinomycin D and etoposide. Free Radic. Res. 44, 864-870.

Yoo, D.Y., Shin, B.N., Kim, I.H., Kim, D.W., Yoo, K.Y., Kim, W., Lee, C.H., Choi, J.H., Yoon, Y.S., Choi, S.Y., et al. (2012). Effects of sensitive to apoptosis gene protein on cell proliferation, neuroblast differentiation, and oxidative stress in the mouse dentate gyrus. Neurochem. Res. 37, 495-502.

Zhu, X., Raina, A.K., Lee, H.G., Casadesus, G., Smith, M.A., and Perry, G. (2004). Oxidative stress signalling in Alzheimer's disease. Brain Res. 1000, 32-39. 